\title{
A 12-COURSE RADIO RANGE FOR GUIDING AIRCRAFT WITH TUNED REED VISUAL INDICATION
}

\author{
By H. Diamond and F. G. Kear
}

ABSTRACT

This paper describes a radio-directive beacon (or radio range), of the visualindicating type, which has been developed by the National Bureau of Standards to provide radio-marked courses at air terminals where more than four airways converge.

The radio range is similar in design to the double-modulation type with the addition of a third amplifier train and accompanying modulation frequency. It provides 12 equisignal zones which may be oriented within rather wide limits and made to coincide with the converging airways.

In order to prevent coupling between amplifier branches special means are employed to supply them successively rather than simultaneously. These are described in detail and the stability of the resulting system is shown to be excellent.

Three-phase radio-frequency supply is used to excite the amplifier trains, which is received from a single-phase oscillator of conventional type by means of a phase divider. This prevents possible trouble due to the employment of a 3 -phase oscillator with 3-tuned oscillating circuits.

To transfer the energy to the antenna system a goniometer with three primary coils is employed. These are displaced $120^{\circ}$ from each other and coupled to the two crossed loop antennas through two secondary coils whose displacement is $90^{\circ}$. This permits the use of two antennas to establish a space pattern from three amplifier branches.

Means for aligning this resultant space pattern with the airways are also discussed and several examples are given.

\section{CONTENTS}

I. Introduction

II. Theory of 12-course radio range. 351

1. Link circuit arrangement

2. Automatic switching using 3-phase grid biasing

3. Three-phase radio-frequency switching

III. Methods of shifting the beacon courses

IV. Tests . . 369

\section{INTRODUCTION}

The rapid increase in the number of airways emanating from the more important airports of the United States has created a need for a directive radiobeacon capable of marking out a greater number of courses than has hitherto been possible. This paper describes a radio range (that is, directive radiobeacon) which provides 12 beacon courses normally disposed $30^{\circ}$ from each other. By means of simple adjustments at the transmitter the angles between courses can be set as desired. This range fulfills all the present requirements for guiding aircraft along the civil airways.

The 12-course radio range is similar in operation to the 2 and 4 course radio ranges previously described. ${ }^{1}$ The increase in apparatus

1 J. H. Dellinger and H. Pratt, Radio Aids to Air Navigation, Proc. Inst. Radio Engrs., 16, pp. 890-920; July, 1928. J. H. Dellinger and H. Diamond, Radio Developments Applied to Aircraft, Mech. Eng., 51, No. 7, pp. 509-514; July, 1929. 


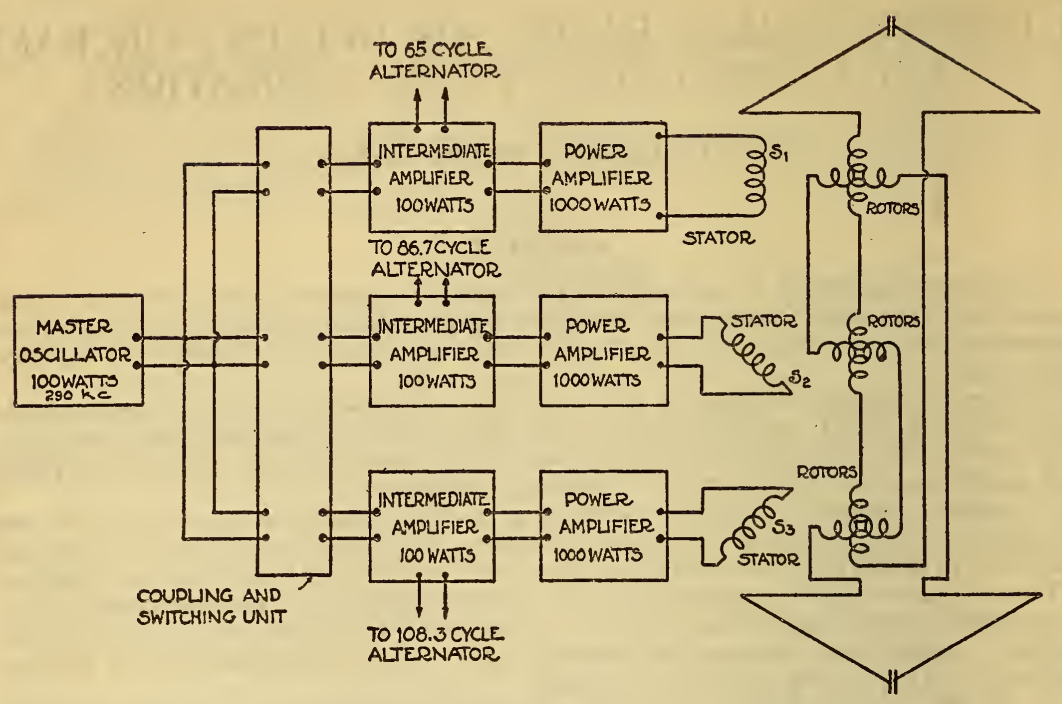

FIGURE 1.-Schematic diagram of 12-course radio range

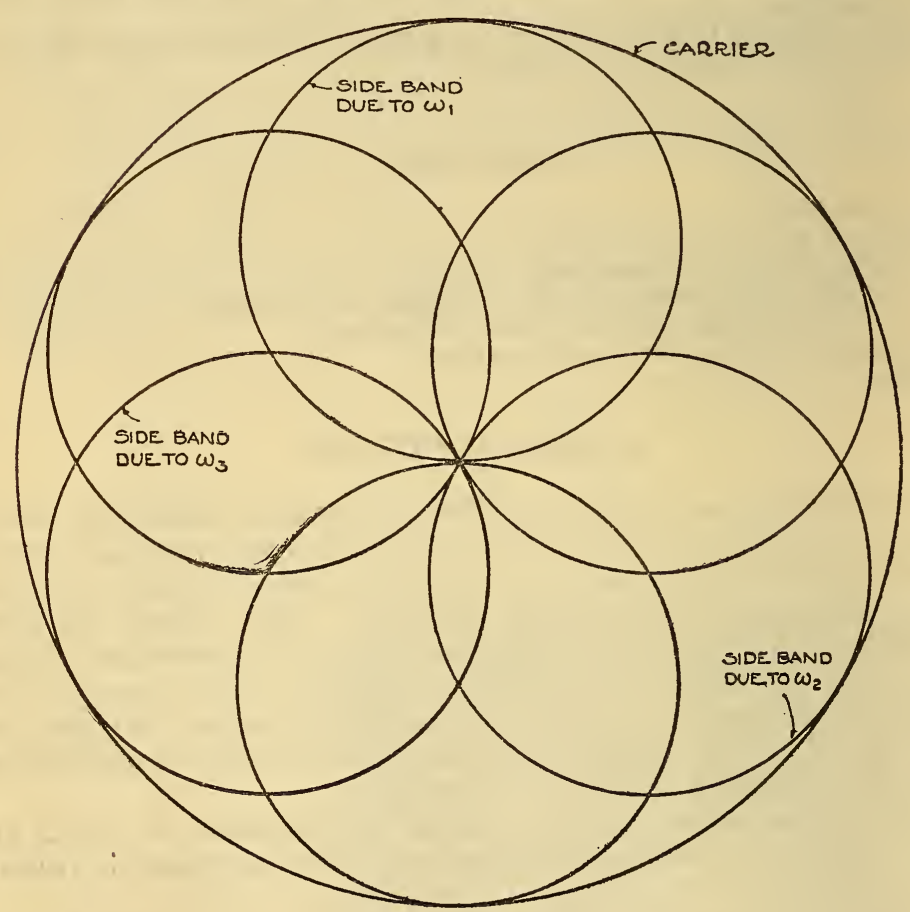

FIGURE 4.-Space pattern radiated by 12-course radio range (link circuit type) 
B. S. Journal of Research, RP154

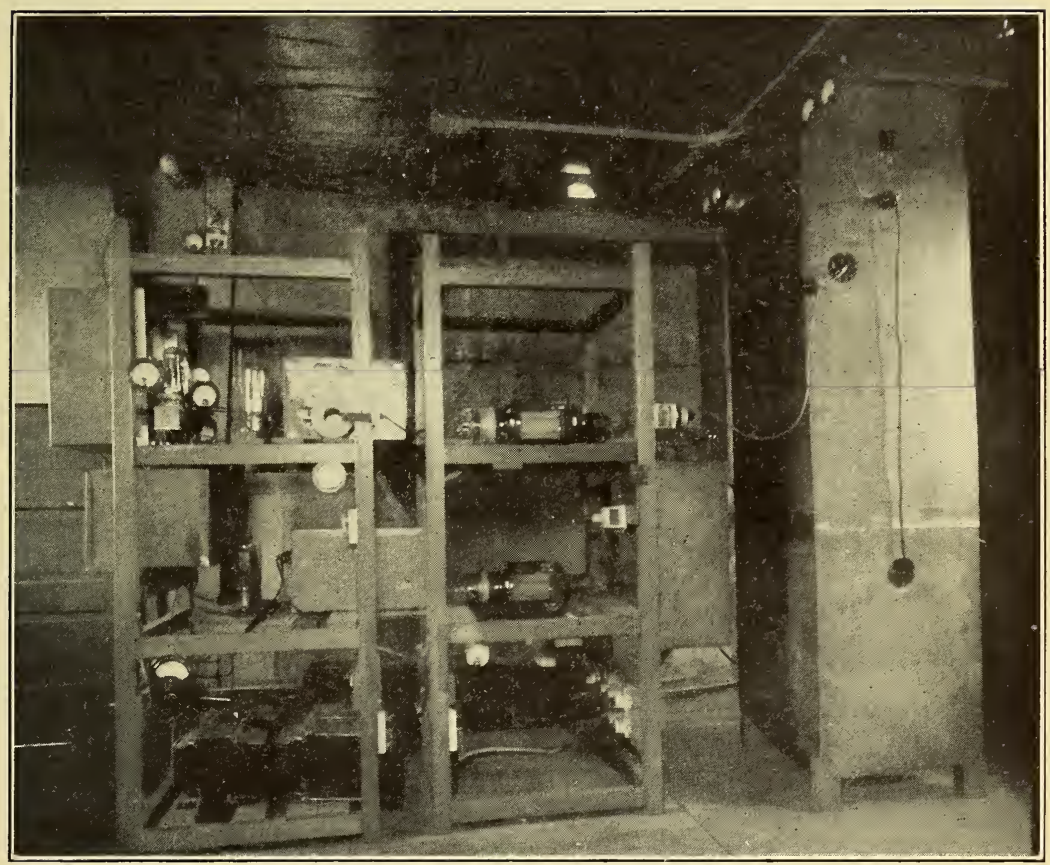

Figure 2.-Experimental 12-course range transmitter installed at College Park, Md. 
B. S. Journal of Research, RP154

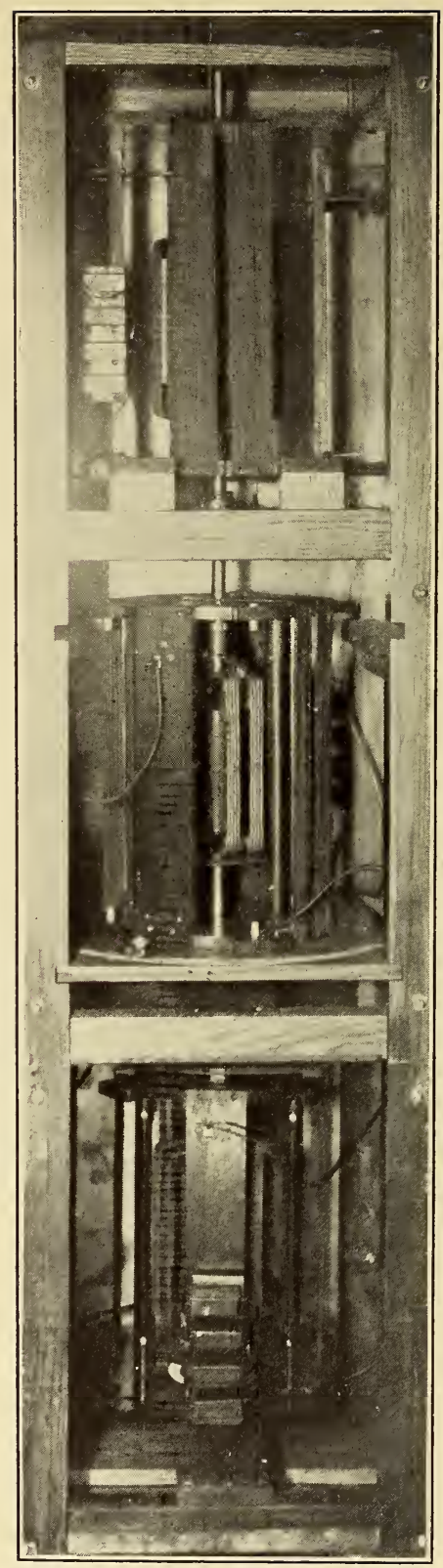

Figure 3.-Special 3-stator goniometer employed with $12-$ course radio range 
is not great. The same crossed-coil antenna system and the same circuit arrangements are employed (see fig. 1), except that three amplifier branches modulated to three different low frequencies are necessary. The modulation frequencies used are $65,86.7$, and 108.3 cycles, respectively. In addition a special goniometer is required. The rotor system of this goniometer comprises two coils crossed at $90^{\circ}$, and each connected in series with one loop antenna. For convenience in goniometer design each rotor coil is made up of three sections. Three stator coils, normally placed at $120^{\circ}$ with each other, are employed, one stator coil being connected to each power amplifier tube of the transmitting system.

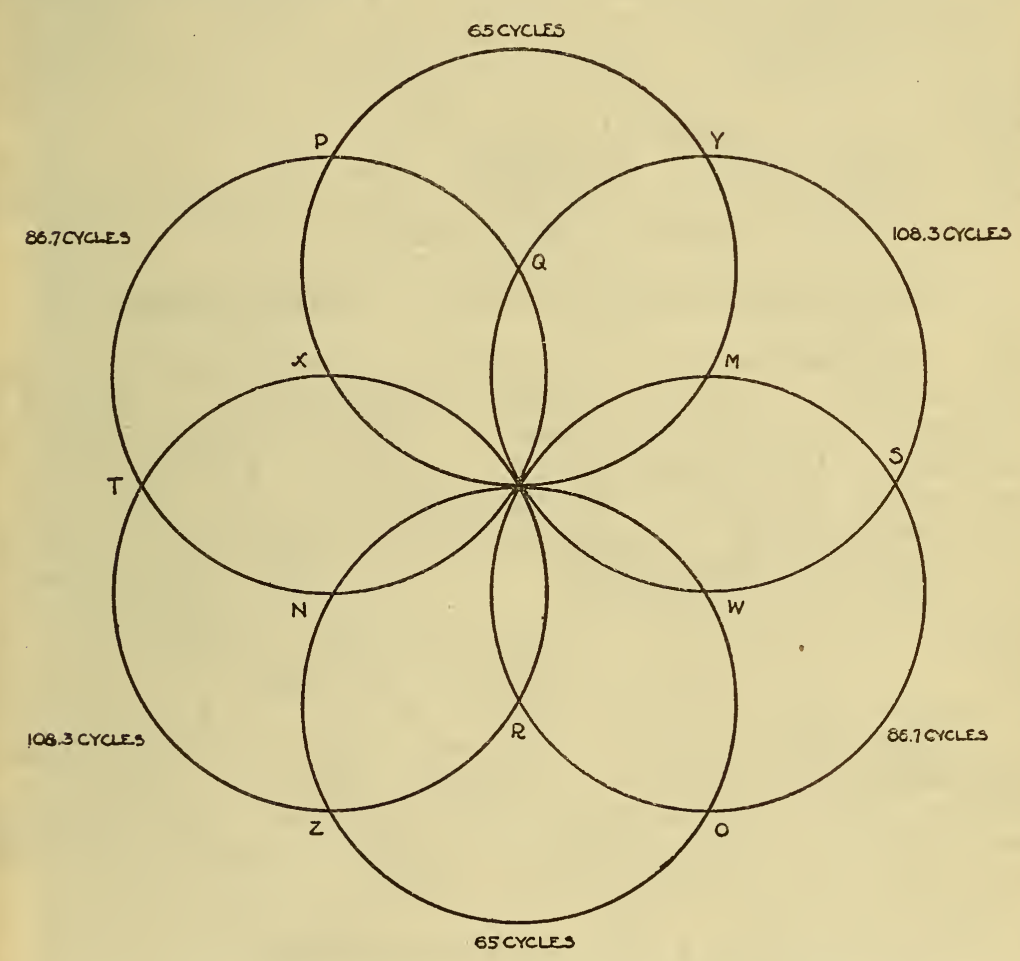

Figure 5.-Polar pattern as received on the reeds

A photograph of the 12-course beacon transmitter installed at College Park, Md., is shown in Figure 2. Figure 3 is a photograph of the special goniometer employed.

Referring to Figure 1, it will be observed that stator coil $S_{1}$ carries a radio-frequency current modulated to 65 cycles, stator coil $S_{2}$ carries a radio-frequency current modulated to 86.7 cycles, and stator coil $S_{3}$ a radio-frequency current modulated to 108.3 cycles. Each stator coil, acting in conjunction with the two crossed rotor coils and the two crossed loop antennas, sets up a system which is electrically equivalent to a single loop antenna. The plane of this phantom antenna coincides with the plane of the stator winding for zero setting of the rotor, but rotates in space as the rotor system is rotated. 
Since there are three stator coils, placed at $120^{\circ}$ with each other, three such phantom antennas (crossed at $120^{\circ}$ ) exist. When special precautions are taken in circuit design the combined space pattern consists of a circular carrier with three figure-of-eight side bands crossed at $120^{\circ}$. (See fig. 4.) The corresponding polar pattern as received on the reeds is shown in Figure 5.

Now assume that a pilot is equipped with three vibrating-reed course indicators of the 2-reed type, ${ }^{2}$ the first tuned to 65 and 86.7 cycles, the second to 86.7 and 108.3 cycles, and the third to 65 and 108.3 cycles, respectively. Using the first indicator, he will find four courses at $M, N, O$, and $P$. With the second course indicator he will observe four courses at $Q, R, S$, and $T$. Similarly, with the third reed indicator, four courses at $W, X, Y$, and $Z$ will be obtained. Two of each set of four courses (for example, $M$ and $N$ ) have an equisignal zone of $1^{\circ}$ to $1.5^{\circ}$ wide, while the width of this zone for the other two courses (viz, $O$ and $P$ ) is from $3^{\circ}$ to $4^{\circ}$.

To simplify the use of this beacon, a special 3-reed indicator for receiving all 12 courses of the beacon without confusion has been developed. ${ }^{3}$ A photograph of this indicator is given in Figure 6.

\section{THEORY OF 12-COURSE RADIO RANGE}

Since the goniometer stator windings are not at $90^{\circ}$ with each other (see fig. 1) undesirable intercoupling between $S_{1}, S_{2}$, and $S_{3}$ will exist, resulting in a combined beacon space pattern which can not be used. Direct inductive coupling between stator windings is eliminated by the use of the 3-section rotor system, each stator winding being placed in a separate shielded compartment. Indirect coupling between stator windings by virtue of their mutual induction with the rotor system still exists. This coupling is somewhat more difficult to eliminate. By setting up the circuit equations for the goniometer system when no precautions are taken to prevent this coupling, and solving for the currents in $S_{1}, S_{2}$, and $S_{3}$, two facts may be determined: (1) That the radio-frequency current in each stator winding is modulated to all three modulation frequencies of the beacon, (2) that the amount of coupling between stator windings is independent of the rotor setting. Several arrangements are possible for preventing this undesirable intercoupling.

\section{LINK CIRCUIT ARRANGEMENT}

One arrangement consists of neutralizing this coupling by introducing inductive coupling between stator windings of opposite sense, as shown in Figure 7. Thus $S_{1}$ is coupled to $S_{2}$ by coils $L_{1} L_{1}^{\prime \prime} L_{2}^{\prime} L_{2}$ and to $S_{3}$ by coils $L_{1} L_{1}^{\prime} L_{3}^{\prime \prime} L_{3}$. Similarly, $S_{2}$ is coupled to $S_{1}$ as noted and to $S_{3}$ by coils $L_{2} L_{2}^{\prime \prime} L_{3}^{\prime} L_{3}$. The link circuits $L_{1}{ }^{\prime \prime} C_{1} L_{2}{ }^{\prime}, L_{2}{ }^{\prime \prime} C_{2} L_{3}{ }^{\prime}$, and $L_{3}{ }^{\prime \prime} C_{3} L_{1}^{\prime}$ are each tuned to the beacon carrier frequency. It is evident that by virtue of these link circuits a current flowing in any stator induces in each of the other stators an e. m. f. $180^{\circ}$ out of phase with that current. On account of the indirect coupling between stators by way of the rotor system a current flowing in any

2 F. W. Dunmore, Design of Tuned-Reed Indicators for Aircraft Radio Beacen, B. S. Jour. Research: November, 1928. (Research Paper No. 28.)

${ }^{3}$ F. W. Dunmore, A Tuned Reed Course Indicator for the 4 and 12 Course Aircraft Radio Range, B. S. Jour. Research. 
B. S. Journal of Research, RPI54

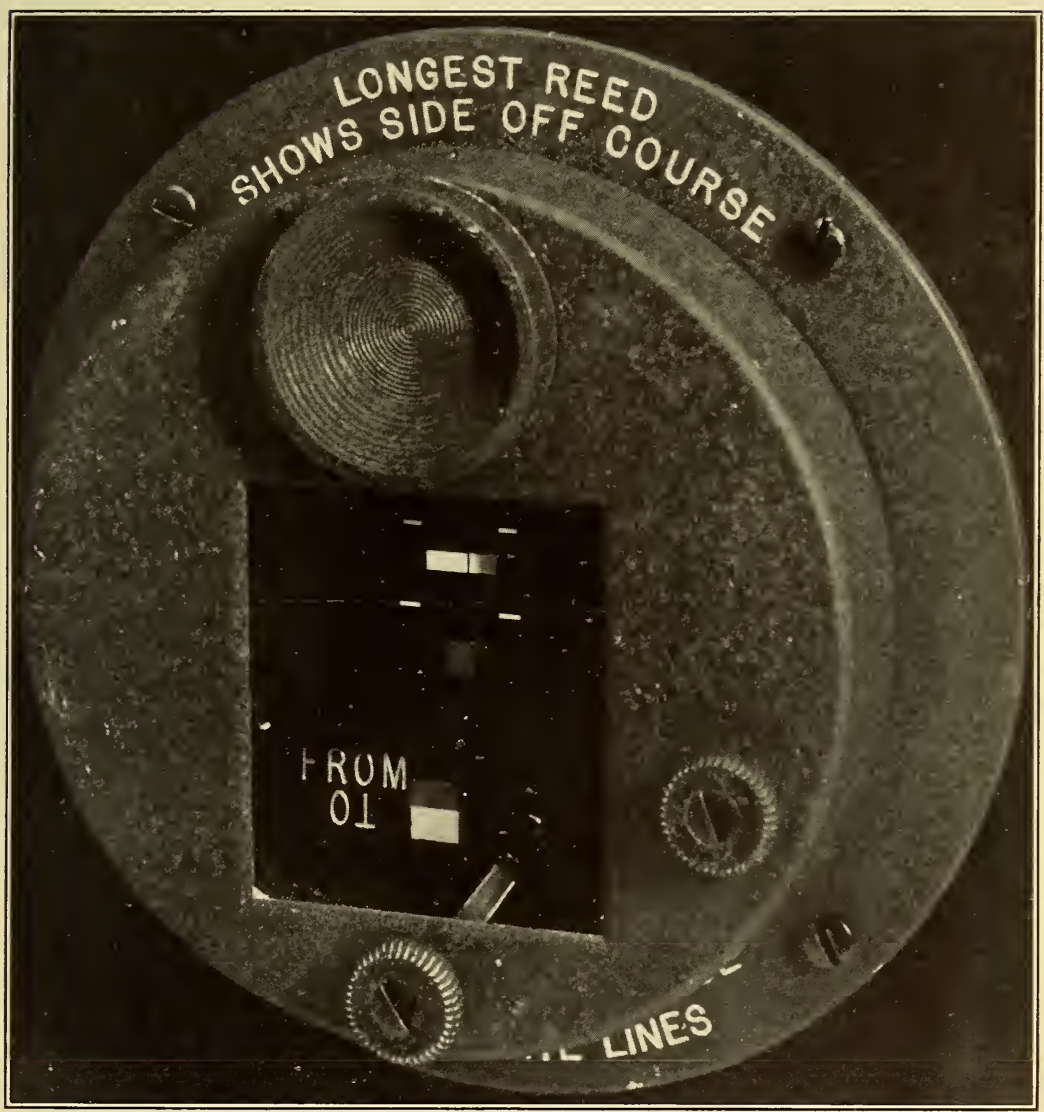

FiguRe 6.-Special course indicator for 12 -course radio range 
one stator induces in each of the other stators a voltage exactly in phase with that current. By properly adjusting the amount of coupling due to the link circuits an exact neutralization may be obtained.

The carrier-frequency currents in $S_{1}, S_{2}$, and $S_{3}$ (fig. 1) have been assumed to be in time phase. Since the stator windings are displaced by $120^{\circ}$ space phase the resultant carrier transmitted is zero. A circular carrier can, however, be supplied by the use of a vertical antenna extending along the beacon tower and coupled through a 50 -watt amplifying tube to the master oscillator of the beacon trans-

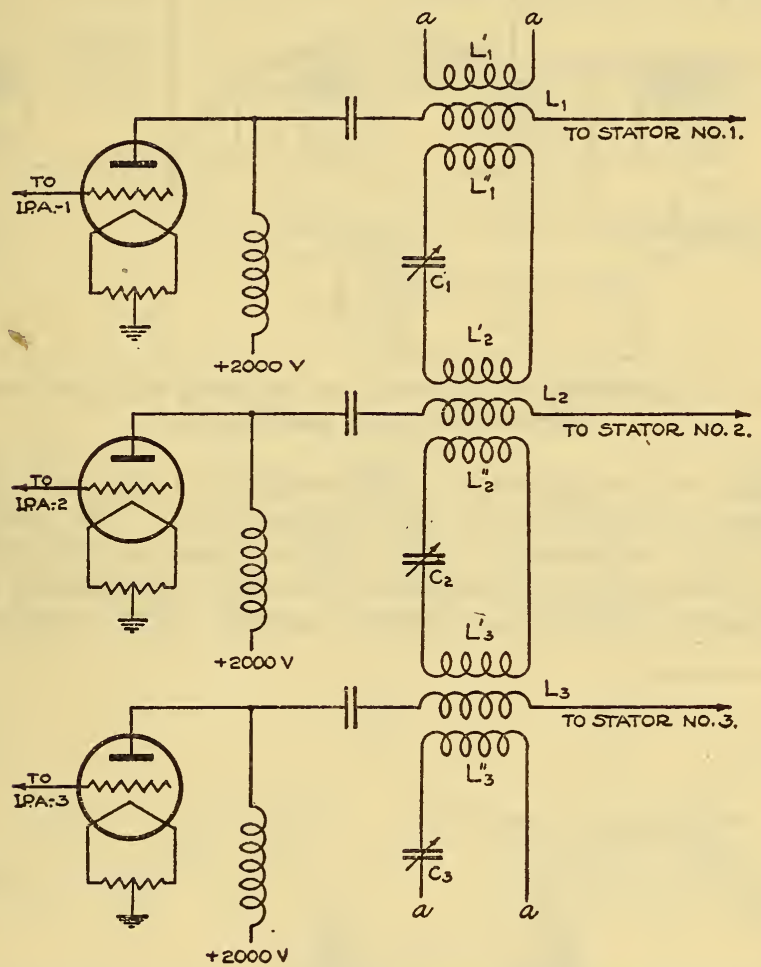

Figdre 7.-Link circuit arrangement for preventing intercoupling between primary goniometer windings

mitting set. For optimum results, this antenna must be in accurate tune to the beacon carrier frequency. The polar patterns shown in Figures 4 and 5 are for this transmitting arrangement.

\section{AUTOMATIC SWITCHING USING 3-PHASE GRID-BIASING}

The use of link circuits to eliminate coupling between stator windings and the need for supplying an auxiliary carrier to replace the one supressed by the goniometer system may both be precluded if means are provided for exciting but one stator winding at a time. The complexity of the scheme outlined above led to experiments toward devising such means. 
An obvious arrangement for accomplishing this consisted of inserting a commutator carrying three segments occupying successive $120^{\circ}$ arcs in the supply leads from the master oscillator to the grids of the intermediate power amplifier tubes. In this

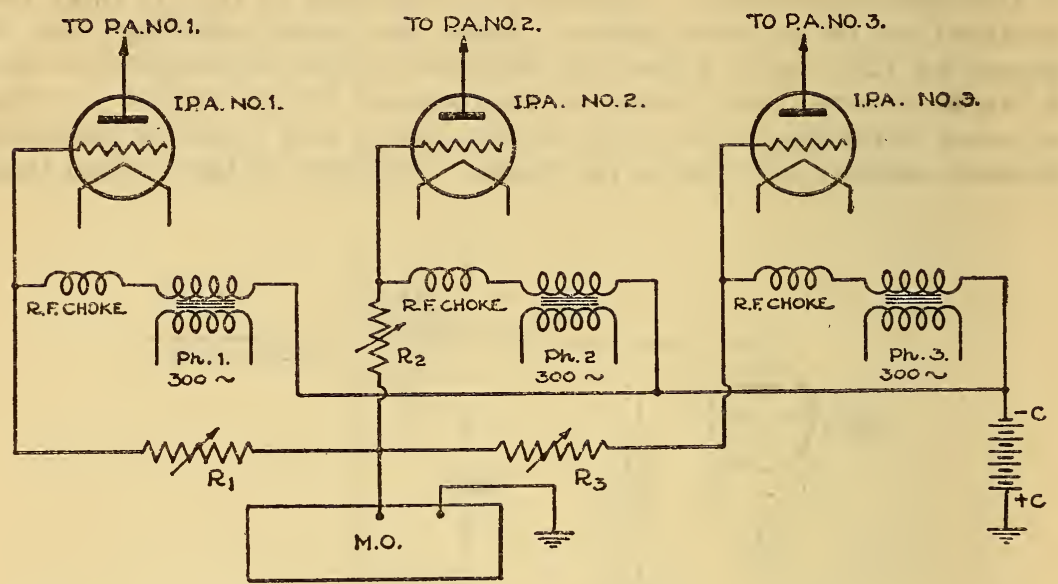

FIGURE 8.-Grid-biasing arrangement for switching radio-frequency supply successively to each amplifier train

way voltage was supplied to each amplifier train only during onethird of each revolution of the commutator. The switching was performed at a sufficiently rapid rate so that in spite of the interruptions in the signals transmitted the reeds maintained their vibration amplitudes due to mechanical inertia, This method had two

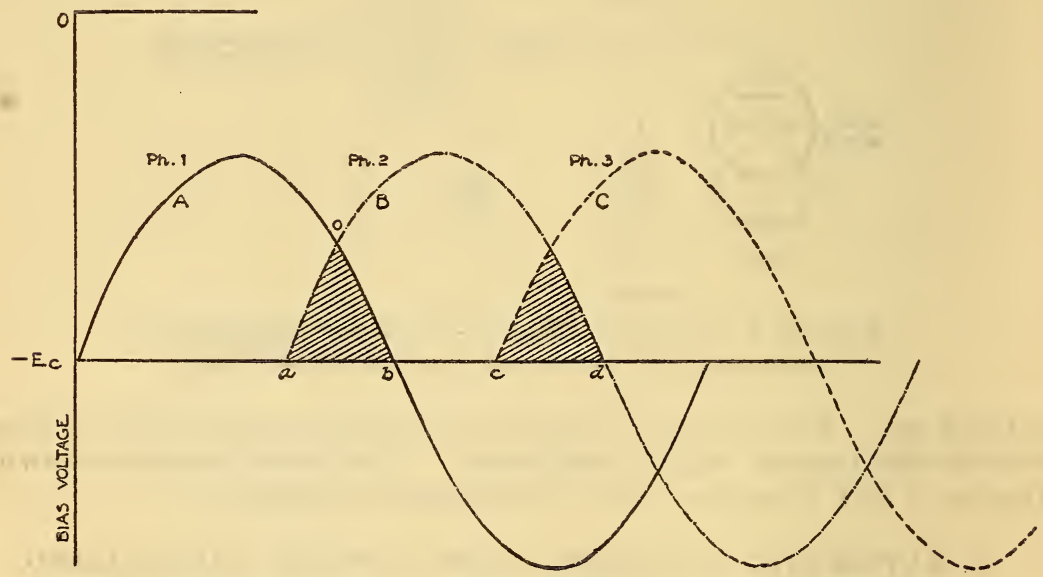

FIGURE 9.-Resultant biasing voltages on grid of each intermediate-amplifier stage

distinct disadvantages: (1) The power transmitted by each amplifier train was reduced to one-third normal, and (2) surges in the transmitted wave due to high-speed switching resulted in shock excitation of the reeds used for reception, the net effect in the beacon operation being a virtual widening of the equisignal zones. 
The next circuit arrangement used proved considerably more successful, the switching of power being performed at an audiofrequency rate by means of the circuit shown in Figure $8 . \mathrm{A} \mathrm{d}$. c. biasing voltage, common to the grid circuits of the three intermediate amplifying tubes, is employed and is of such magnitude that (in the absence of the 3-phase 300-cycle supply) no power is transmitted through these tubes. In series with this common d. c. biasing voltage, each grid circuit has induced in it one of the phase voltages of the 3-phase 300-cycle supply. The resultant biasing voltages impressed on the grid of each of the three intermediate amplifying tubes are indicated by curves $A, B$, and $C$, respectively, of Figure 9. Since $-E_{c}$ is the cut-off voltage, each tube passes

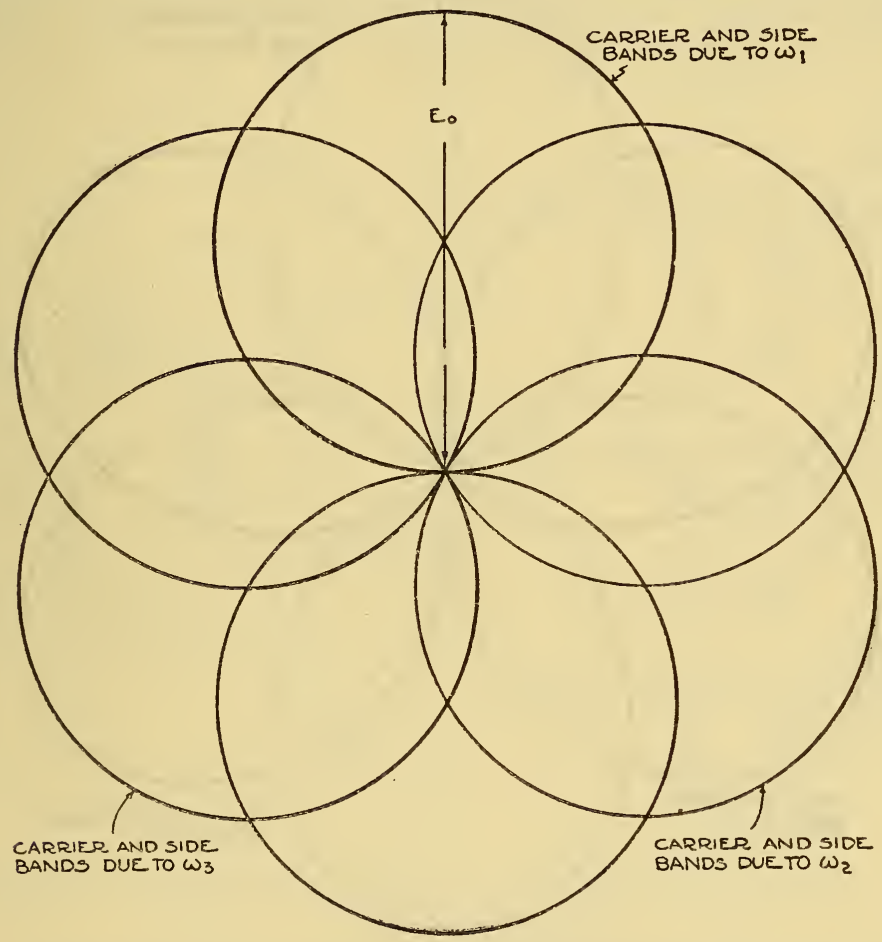

FIGURE 10.- Space pattern radiated by beacon when using grid biasing arrangement for preventing intercoupling between primary goniometer windings

power only during the positive half-cycle of its a. c. biasing voltage. As a result no two amplifier tubes transmit power simultaneously except during the small intervals of time $a-b, c-d$, etc., as shown in Figure 9 . The proportion of power transmitted during these intervals is less than the ratio of shaded area $a o b$ to total area of transmission per cycle for one tube $E_{c} a o b$, since the tube is then operating on the knee of its characteristic curve. The amount of coupling still present may be further reduced by increasing, the common $\mathrm{d}$. c. bias voltage, but this results also in a decrease of total power transmitted. In practice this further reduction is unnecessary, the beacon performance proving satisfactory without it. 
Neglecting the small amount of coupling present the beacon space pattern becomes as shown in Figure 10 . Note that, since but one stator winding is excited at a time, there are three independent carrier waves in the beacon-space pattern. Assuming square-law detection the polar pattern as received on the reeds is shown in Figure 11.

The choice of frequency of the 3-phase alternator used for the grid bias switching is important. The fundamental and second harmonic of this frequency must not be closer than approximately 20 cycles to any of the harmonics of the modulation frequencies used in the beacon. To illustrate the reason for this requirement

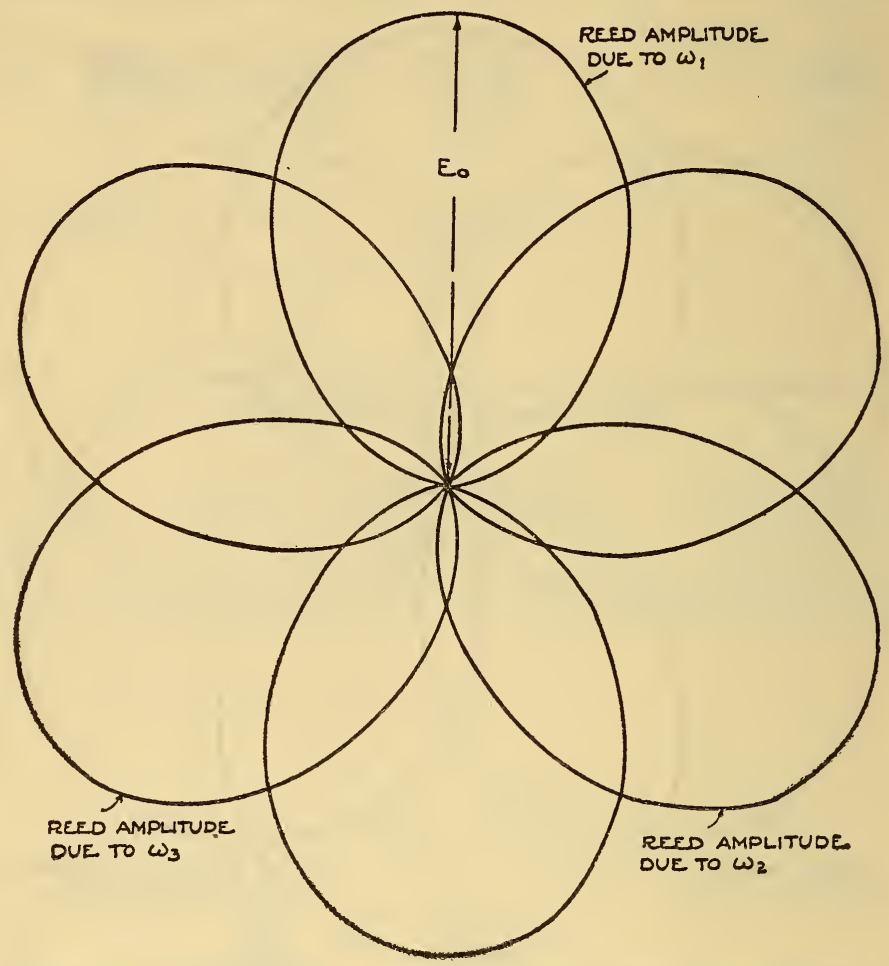

Figure 11.-Received pattern corresponding to space pattern of Figure 10

assume that a frequency of 262 cycles were chosen for the 3-phase alternator. The fourth harmonic of 65 cycles and the third harmonic of $86 \%$ cycles are each 260 cycles. Under this condition a beating of the reed indicator at the difference frequency of 2 cycles is obtained.

The net power transmitted by each amplifier branch when using this method of switching is, of course, reduced considerably below normal; primarily because power is transmitted only during approximately one-half the time, and secondly because during that time the operating point travels along the tube characteristic from the point of cut-off to the point of normal operation and back to the cut-off point. The net reduction in power is, however, not as great as might be expected from the above, since it is possible to force the tubes con- 
siderably above normal during the half cycle of operation and still maintain safe average plate dissipation. Using this system, with 8 amperes in each loop antenna, an operating range of 100 miles was obtained.

\section{THREE-PHASE RADIO-FREQUENCY SWITCHING}

The use of 3-phase audio-frequency for switching the radio-frequency power as described above suggested that the same results could be obtained if a source of 3-phase radio-frequency were available. Each phase of this 3-phase radio-frequency supply could then be used for supplying carrier voltage to the grid of one intermediate amplifier, the 3-phase source thus serving as a master oscillator as well as a switching device. The advantages to be gained are fourfold:

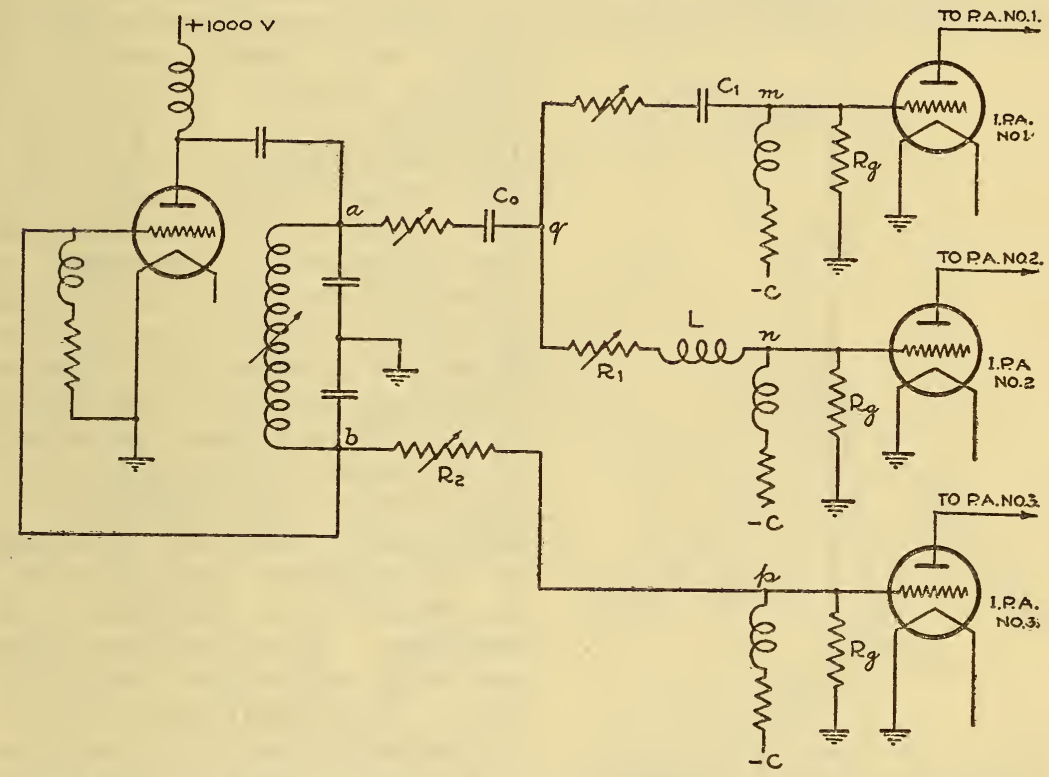

FIGURE 12.-Phase-splitting arrangement for obtaining 3-phase radiofrequency switching

(a) A decided increase in the power transmitted by each amplifier branch, $(b)$ the elimination of a 3-phase audio-frequency unit, $(c)$ the operation of the intermediate amplifier tubes under normal conditions and not with the high grid voltages necessary in the method of grid bias switching, and $(\bar{d})$ the elimination of interference caused by the audio-frequency used for grid biasing.

A 3-phase radio-frequency oscillator of the type described by $R$. Mesny ${ }^{4}$ was constructed to serve as the 3 -phase source. However, advantages of simplicity in design and operation led to the development of the phase-splitting arrangement shown in Figure 12.

Referring to Figure 12, a single-phase Colpitts oscillating circuit is employed. Advantage is taken of the fact that the voltages from

${ }^{4}$ R. Mesny, Generation of Polyphase Oscillations by Means of Electron tubes, Proc. I. R. E., 13, pp. 471-476; August, 1925. 
points $a$ and $b$ to ground are approximately $180^{\circ}$ out of time phase. It is desired that the voltages from points $m, n$, and $p$ to ground have a $120^{\circ}$ time-phase displacement. The values of the condenser, $C$, and the inductance, $L$, are so chosen that the voltage from $m$ to ground leads the voltage from $q$ to ground by $60^{\circ}$ and the voltage from $n$ to ground lags the same voltage by $60^{\circ}$. The condenser, $C_{0}$, is so chosen that the voltage from ' $q$ to ground is $180^{\circ}$ out of phase with the voltage from $p$ to ground. The variable resistances shown aid materially in making these adjustments. The impedances of the various branches must be such that the voltages from $m, n$, and $p$ to ground are equal in magnitude as well as $120^{\circ}$ out of phase. The voltage vector diagram corresponding to this adjustment is shown in Figure 13.

As will be explained below, the time-phase displacement between the voltages applied to the grids of the three intermediate amplifying:

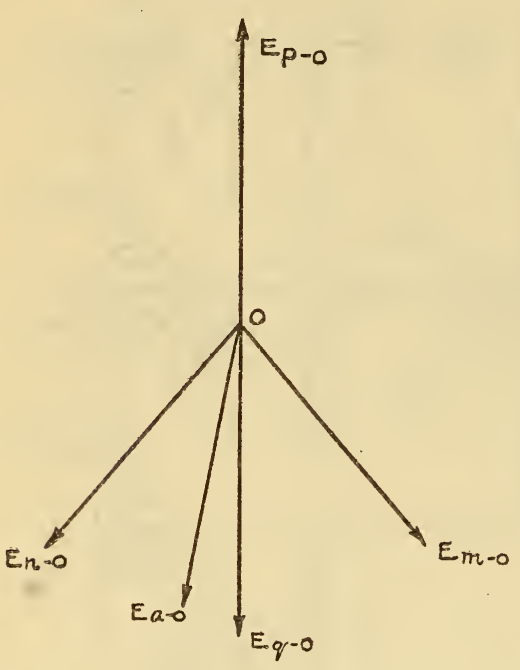

Frgure 13.-Voltage vector diagram corresponding to circuit of Figure 12 tubes must remain constant to prevent a shifting of the beacon courses in space. A variation of the grid to filament tube impedances would tend to cause such a displacement. The resistances, $R_{g}$ (having values of $7,500 \mathrm{ohms}$ ) are connected in parallel with the grid to filament impedances to minimize such variation. With this arrangement, a 20 per cent change in the grid to filament impedance of any tube results in but a $2^{\circ}$ variation in the time-phase displacement. Similarly, a change in the oscillator frequency of 2 kc. results in a $0.2^{\circ}$ time-phase variation. As will be shown, these are well within the permissible limits. The means employed for adjusting the voltages from points $m$, $n$, and $p$ to ground to their proper phase relationship is of interest.

The use of $7,500-\mathrm{ohm}$ resistors in parallel with the tube input impedance has already been mentioned. With these resistors in use, the load impedances of the phase divider remain sensibly constant and adjustments can be made on that basis.

First, condensers $C_{0}$ and $C$ are short-circuited and the output of the master oscillator is adjusted by raising or lowering its plate voltage until the grid meter in the branch mo reaches a convenient reading, $I_{0}$. The current in branch no will then read, say $I_{1}$. Condenser $C$ is then inserted in the circuit and its capacity adjusted until the current in the circuit mo is reduced to one-half of its former value. At the same time the master oscillator output is kept constant by keeping the grid current in branch no at $I_{1}$. When the current is halved, assuming a purely resistive circuit in the first instance, the phase angle of the voltage mo has been adjusted to a $60^{\circ}$ leading angle with the voltage $q o$. 
Next, with $C_{0}$ still short-circuited, the process is repeated in the branch no, adjustment of $L$ being made until the desired angle is secured. At the same time the resistance $R_{1}$ is varied to keep the total circuit impedance the same as that of the condenser branch. This is readily noted from the grid milliammeter readings, which should read the same in both branches.

The voltages mo and no are now in the correct phase relationship. There remains the selection of the proper value for $C_{0}$ in order that qo be in $180^{\circ}$ time phase with po. This adjustment is not as straightforward as the preceding ones.

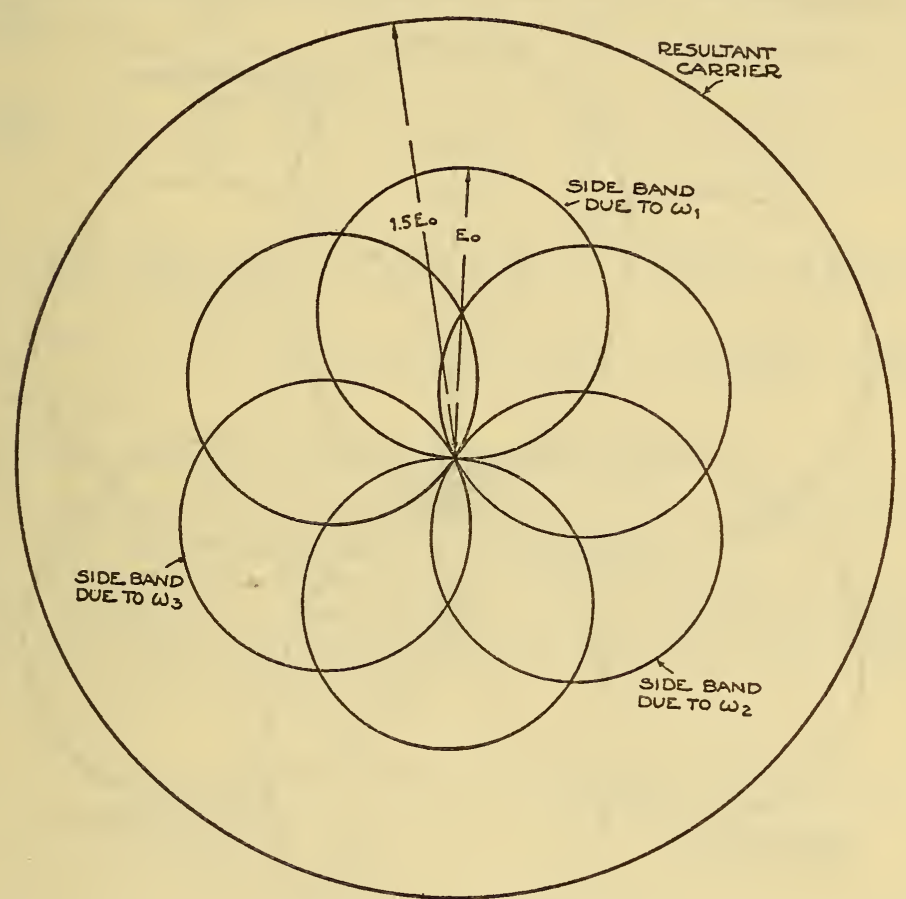

FIGURE 14.-Beacon space pattern when using radio-frequency

As previously explained, when the phase relations are correct, the carrier may be considered as a rotating figure-of-eight of constant

magnitude. This effect is utilized in making the final adjustment.
With $d$. c. excitation on the plates of the intermediate power amplifier tubes and the condenser $C_{0}$ in the circuit, the output of each amplifier branch is separately balanced in magnitude by adjustment of $R_{2}$ until all three are equal as shown by the antenna currents. goniometer rotated. When $C_{0}$ is of the correct value the antenna current remains constant for all positions of the rotor. $C_{0}$ is, consequently, varied until this constancy of antenna current occurs. A readjustment of $R_{2}$ is necessary after each change of $C_{0}$ in order that the magnitudes of the three voltages remain equal. The three voltages are now in correct time phase and will not change appreciably 
with changes in amplifier load or tube constants encountered in normal operation.

There is one important difference between the radio-frequency method of switching and the audio-frequency grid bias method previously described. In the latter method coupling between stator windings was eliminated because the stators were never excited simultaneously. In the radio-frequency method the coupling is eliminated chiefly due to the time-phase displacement.

The beacon space pattern obtained when using this system and the corresponding received polar diagram are shown in Figures 14 and 15 , respectively. Note that even for the same power transmitted

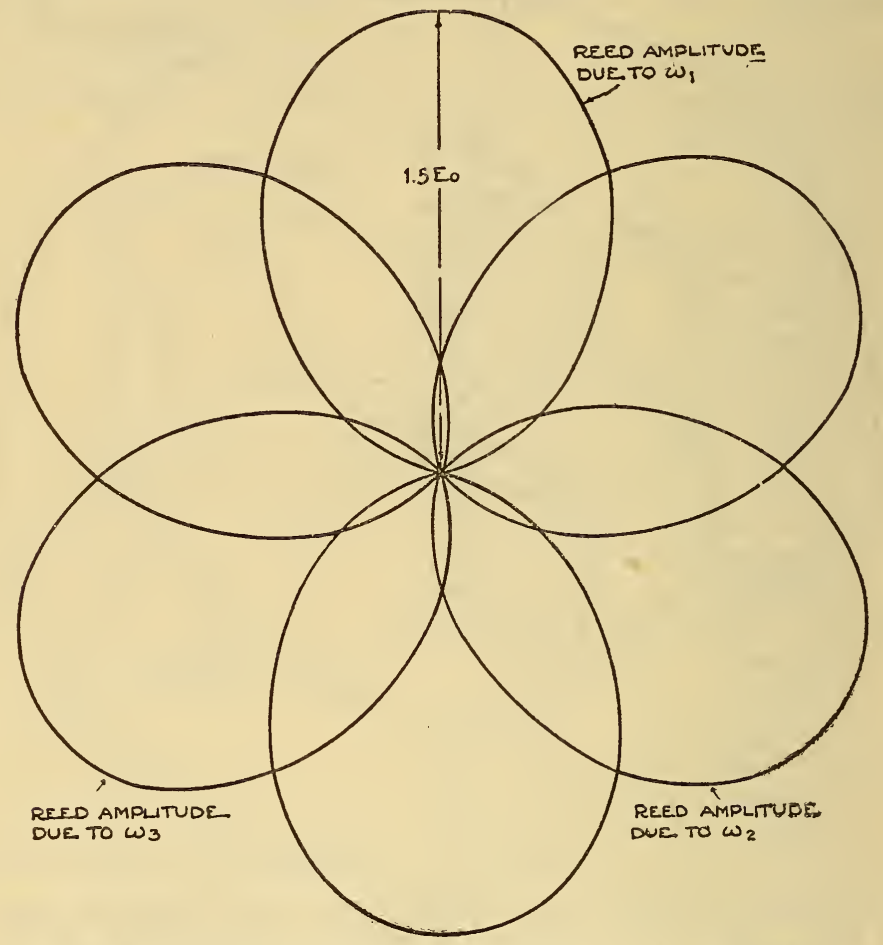

FIGURE 15.-Received pattern corresponding to space pattern of Figure 14

by each amplifier branch this system yields 50 per cent greater received signal than the system employing grid-bias switching. The patterns of Figures 14 and 15 are for the special case when the three stator windings are exactly $120^{\circ}$ apart in space phase and the three voltages applied to these stators exactly $120^{\circ}$ apart in time phase. The general expression for the space pattern for any time phase relationship between these three voltages is given in equation (1). The first term to the right of the equality sign represents the carrier, while the second, third, and fourth terms represent the side bands due to modulation frequencies, $\omega_{1}, \omega_{2}$, and $\omega_{3}$, respectively. As will be observed, the carrier comprises a figure of eight revolving in space at the carrier-frequency rate. The curve representing the carrier in 
Figure 14 is in reality the locus in space of successive maxima of the rotating figure.

$$
\begin{aligned}
e_{p} & =K E_{0}\left\{\begin{array}{c}
\cos \left(\omega t+\Delta_{1}\right) \cos \theta+\cos \left(\omega t-\frac{2 \pi}{3}+\Delta_{2}\right) \cos \left(\theta-\frac{2 \pi}{3}\right) \\
+\cos \left(\omega t-\frac{4 \pi}{3}+\Delta_{3}\right) \cos \left(\theta-\frac{4 \pi}{3}\right)
\end{array}\right\} \\
& +\frac{K E_{1}}{2}\left\{\cos \left(\omega t+\Delta_{1}-\omega_{1} t\right)+\cos \left(\omega t+\Delta_{1}+\omega_{1} t\right)\right\} \cos \theta \\
& +\frac{K E_{2}}{2}\left\{\cos \left(\omega t-\frac{2 \pi}{3}+\Delta_{2}-\omega_{2} t\right)+\cos \left(\omega t-\frac{2 \pi}{3}+\Delta_{2}+\omega_{2} t\right)\right\} \cos \left(\theta-\frac{2 \pi}{3}\right) \\
& +\frac{K E_{3}}{2}\left\{\cos \left(\omega t-\frac{4 \pi}{3}+\Delta_{3}-\omega_{3} t\right)+\cos \left(\omega t-\frac{4 \pi}{3}+\Delta_{3}+\omega_{3} t\right)\right\} \cos \left(\theta-\frac{4 \pi}{3}\right)
\end{aligned}
$$

where

$\left(e_{p}\right.$ is the field intensity at any point, $P$, in space as a polar function of the angle $\theta$.

$\frac{E_{1}}{E_{0}} \times 100$ is the percentage modulation in amplifier branch 1 due to $\omega_{1}$

$\frac{E_{2}}{E_{0}} \times 100$ and $\frac{E_{3}}{E_{0}} \times 100$ have similar meanings.

$\Delta_{1}, \Delta_{2}$, and $\Delta_{3}$, are the time-phase displacement angles of the three carrier components from exact $120^{\circ}$ relationship.

The corresponding expression for the received polar pattern is given by equation (2), assuming square-law detection. The three terms to the right of the equality sign represent the reed amplitudes due to $\omega_{1}, \omega_{2}$, and $\omega_{3}$, respectively.

$$
e_{r_{0}}=K K^{\prime}\left[\begin{array}{l}
E_{0} E_{1} \cos \omega_{1} t\left\{\cos ^{2} \theta+\cos \alpha_{1} \cos \theta \cos \left(\theta-\frac{2 \pi}{3}\right)+\cos \alpha_{3}\right. \\
\left.\cos \theta \cos \left(\theta-\frac{4 \pi}{3}\right)\right\} \\
+E_{0} E_{2} \cos \omega_{2} t\left\{\cos ^{2}\left(\theta-\frac{2 \pi}{3}\right)+\cos \alpha_{1} \cos \theta \cos \left(\theta-\frac{2 \pi}{3}\right)\right. \\
\left.+\cos \alpha_{2} \cos \left(\theta-\frac{2 \pi}{3}\right) \cos \left(\theta-\frac{4 \pi}{3}\right)\right\} \\
+E_{0} E_{3} \cos \omega_{3} t\left\{\cos ^{2}\left(\theta-\frac{4 \pi}{3}\right)+\cos \alpha_{3} \cos \theta \cos \left(\theta-\frac{4 \pi}{3}\right)\right. \\
\left.\quad+\cos \alpha_{2} \cos \left(\theta-\frac{2 \pi}{3}\right) \cos \left(\theta-\frac{4 \pi}{3}\right)\right\}
\end{array}\right]
$$

where $e_{\tau}$ is the signal received on the reeds as a polar function of the angle $\theta$.

$$
\alpha_{1}=\frac{2 \pi}{3}+\Delta_{1}-\Delta_{2}
$$




$$
\begin{aligned}
& \alpha_{2}=\frac{2 \pi}{3}+\Delta_{2}-\Delta_{3} \\
& \alpha_{3}=\frac{2 \pi}{3}+\Delta_{3}-\Delta_{1}
\end{aligned}
$$

Reference to Figure 16 will show that $\alpha_{1}, \alpha_{2}$, and $\alpha_{3}$ are, respectively, the time-phase displacement in degrees between the three carrier voltage vectors of the transmitting system. The amplitudes of these voltage vectors are in turn space functions of the angle $\theta$. It should be noted that each set of side bands transmitted by one amplifier branch beats with its own carrier and also with the in-phase components of the other two carriers of the system.

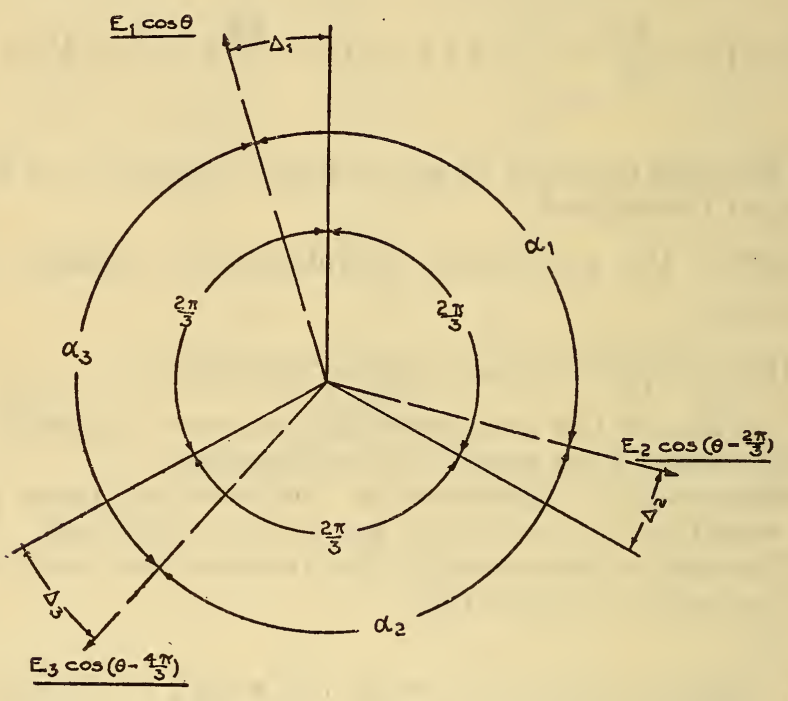

FIGURE 16.-Graphical interpretation of equation (2)

As noted above, a departure of the three carrier voltages from exact $120^{\circ}$ time-phase displacement results in a shifting of the beacon courses in space. The extent of this shift can be determined by substituting special values for the time-phase displacements in equations (1) and (2) above.

For the conditions

$$
\begin{aligned}
& \alpha_{1}=120^{\circ} \\
& \alpha_{2}=150^{\circ} \\
& \alpha_{3}=90^{\circ}
\end{aligned}
$$

which is equivalent to a $30^{\circ}$ time-phase displacement of one of the voltage vectors, the beacon space pattern becomes as shown in Figure 17 and the received pattern as shown in Figure 18. Note that the maximum shift of any course from its normal position is $10^{\circ}$. As noted above, the maximum time-phase variation to be expected, due to variations in the circuit constants, is but $2^{\circ}$. The beacon courses will therefore remain practically fixed in space. 


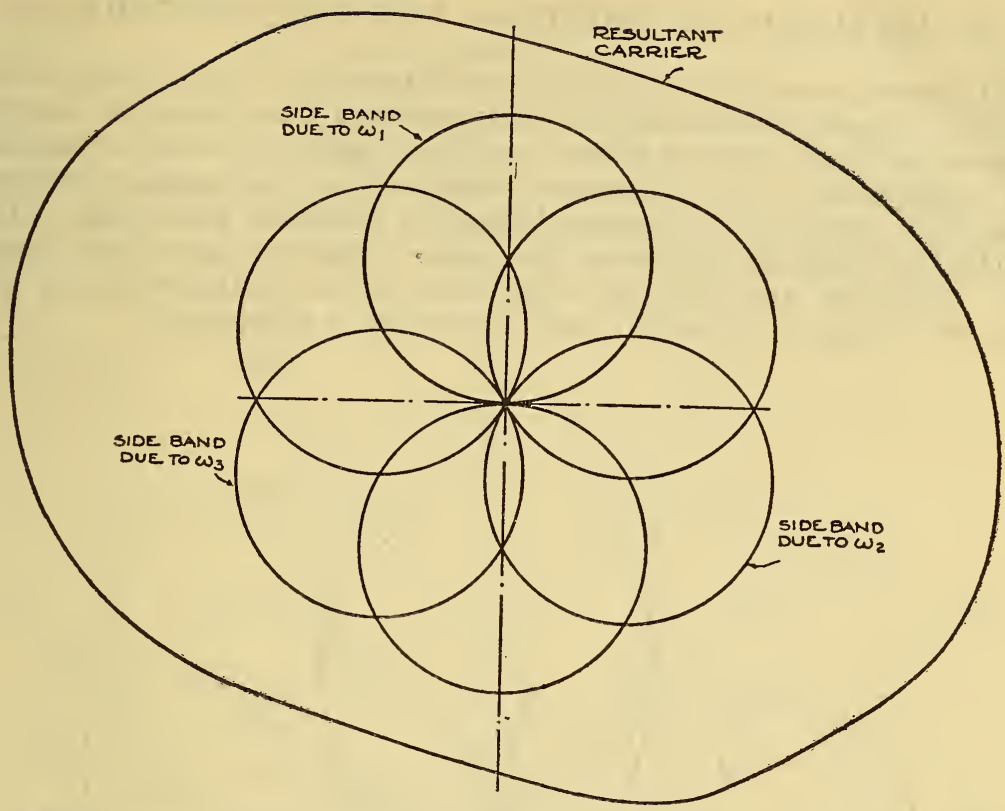

FIGURE 17.-Beacon space pattern when the time phase of one of the three carrier-frequency voltages of the system is displaced by $30^{\circ}$ from its nor-
mal value

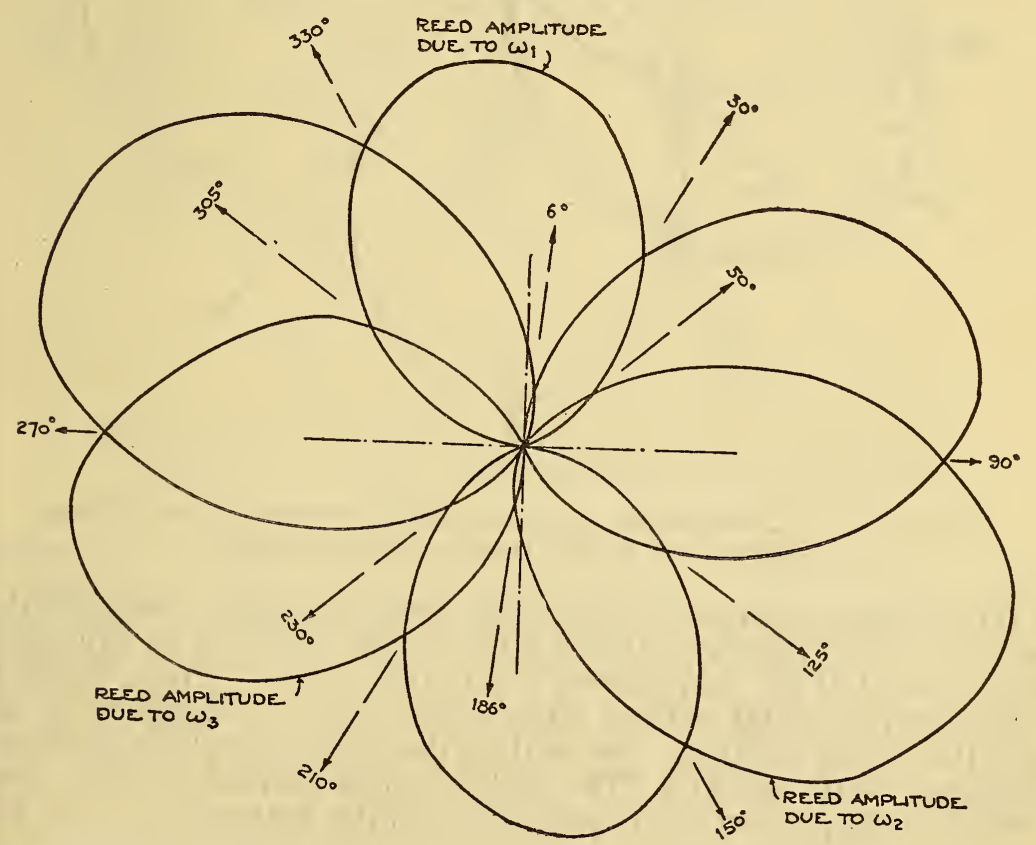

FIGURE 18.-Received pattern corresponding to space pattern of Figure 17 


\section{METHODS OF SHIFTING THE BEACON COURSES}

The same means are available for shifting the beacon courses from their $30^{\circ}$ space relationship (in order to align them with the airways emanating from a given airport) as have been previously described for application to the 4-course radio range. ${ }^{5}$ A simpler method, applicable only to the 12-course beacon is, however, preferable. This method consists of displacing the stator windings from their normal $120^{\circ}$ positions, and may be used when either audio-frequency grid bias switching or radio-frequency switching is employed.

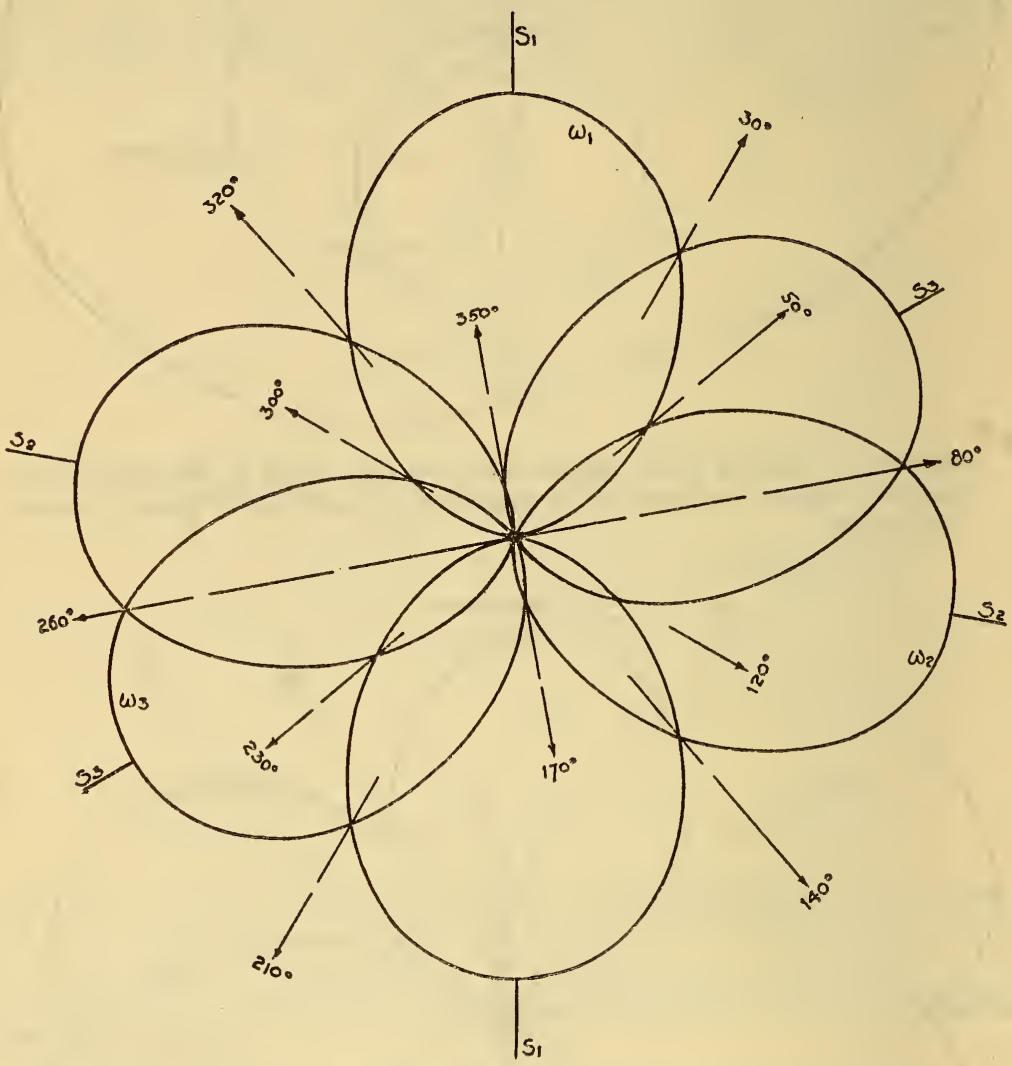

Figure 19.-Displacement of beacon courses obtained when shifting stator No. 2 by $20^{\circ}$ from its normal position

When audio-frequency switching is used, the stators being excited one at a time, displacement of a given stator results in an equivalent displacement of the field pattern due to that stator. Thus, Figure 19 is the received polar pattern when stator No. 2 is displaced by $20^{\circ}$ from its normal position. The pattern due to $S_{2}$ is similarly displaced. (Compare with fig. 11.) The eight courses formed by the intersection of the pattern due to stator $S_{2}$ with the patterns due to $S_{1}$ and

${ }^{5}$ H. Diamond, Applying the Visual Double-Modulation Type Radio Range to the Airways, B. S. Jour: Research. 
$S_{3}$ are all shifted by $10^{\circ}$ in the direction of displacement of $S_{2}$, while the four courses due to the patterns of $S_{1}$ and $S_{3}$ remain fixed in their normal positions. A greater variation of the angles between courses may be obtained by displacing two of the three stators in equal amounts, but in opposite directions. Figure 20 corresponds to a case of this kind, $S_{2}$ and $S_{3}$ being separated from $S_{1}$ by $130^{\circ}$ instead of the normal value of $120^{\circ}$. The four courses formed by the intersection of the patterns due to $S_{1}$ and $S_{2}$ are here rotated $5^{\circ}$ clockwise.

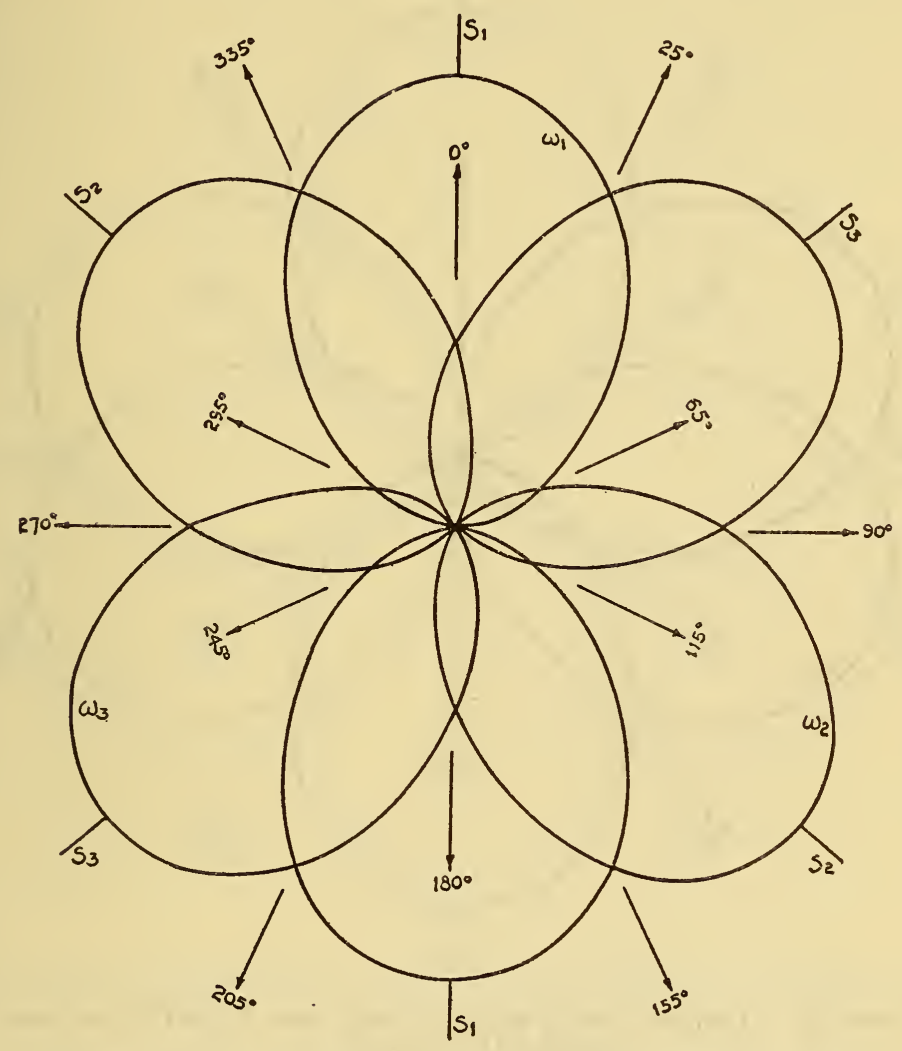

Figure 20.-Displacement of beacon courses when $S_{2}$ and $S_{3}$ are separated by $130^{\circ}$ from $S_{1}$ instead of the normal value of $120^{\circ}$

The four courses due to the patterns of $S_{1}$ and $S_{3}$ are rotated $5^{\circ}$ counter clockwise, while the four courses due to $S_{2}$ and $S_{3}$ remain fixed. Figure 21 is for the case when $S_{2}$ and $S_{3}$ are separated from $S_{1}$ by $110^{\circ}$. In this case the four courses due to $S_{1}$ and $S_{2}$ are rotated $5^{\circ}$ counterclockwise, the four courses due to $S_{1}$ and $S_{3}$ are rotated $5^{\circ}$ clockwise, and the four courses due to $S_{2}$ and $S_{3}$ again remain fixed. Because of the change in direction of rotation the angles between courses are quite different from those obtained in Figure 20. Note also that the majority of the courses are somewhat more useful in so far as signal strength and sharpness of course are concerned. 
Using this method of attack it becomes possible to align the beacon courses with the airways at a great number of airports. In certain special cases, however, it may become necessary to resort to the other methods of course shifting described in the previous paper. ${ }^{6}$

In the system using radio-frequency switching of power, the fact that displacing the stators from their normal position serves to dis-

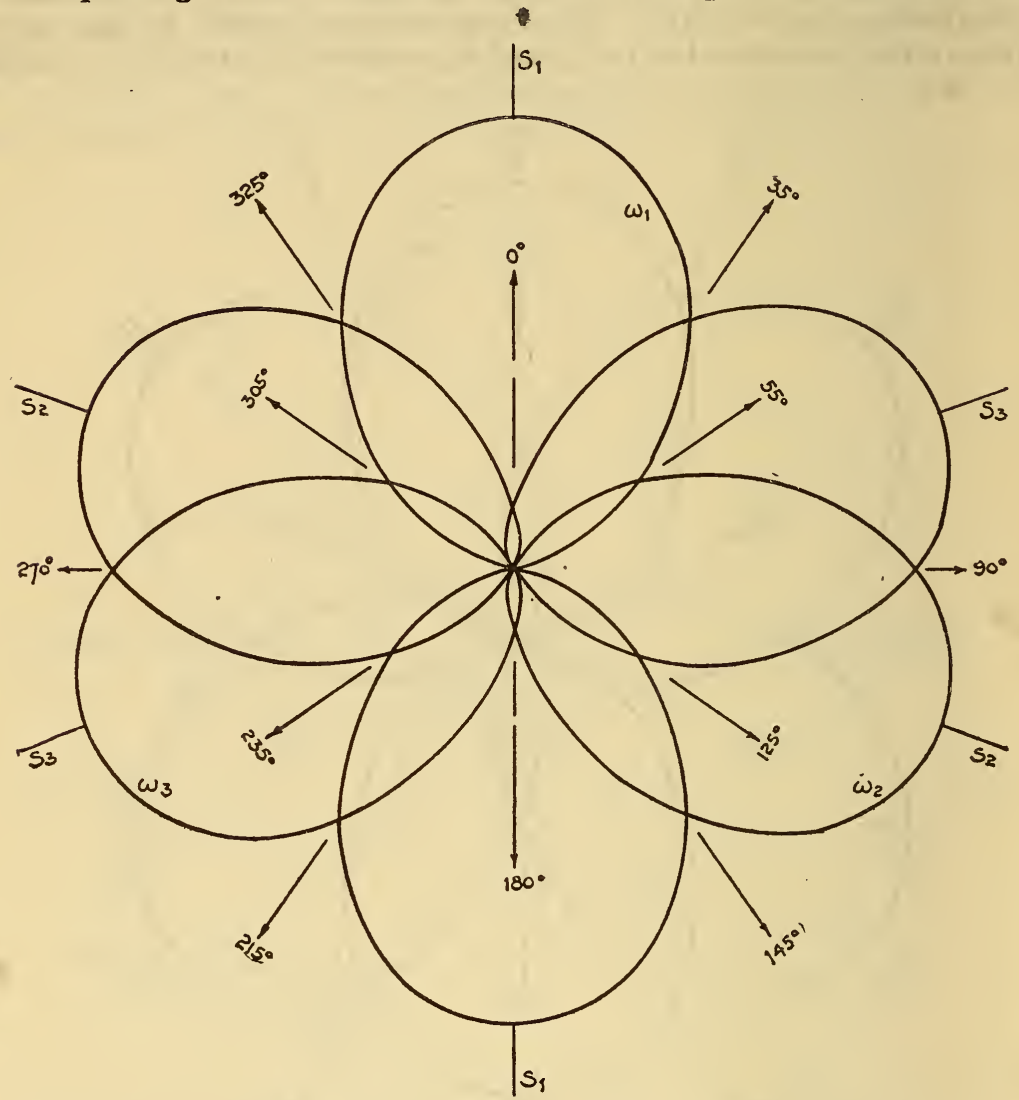

Figure 21.-Displacement of beacon courses whon $S_{2}$ and $S_{3}$ are separated by $110^{\circ}$ from $S_{1}$

tort the carrier renders this method of course shifting a little less powerful. Figure 22 is for the case when $S_{2}$ and $S_{3}$ are separated from $S_{1}$ by $110^{\circ}$. Note that the variation of the courses from their normal positions is not exactly the same as for Figure 21. Enough variation can be obtained with this method, however, to meet the requirements at most airports. 


\section{TESTS}

Numerous test flights have been made on the 12-course beacon described above. The distances obtainable on all 12 courses have been determined. Under average daytime conditions a distance of 100 miles on the weak courses and a correspondingly greater range on the strong courses is obtained. Ground tests were made at Media, Pa., 105 miles from College Park, Md., all 12 courses being

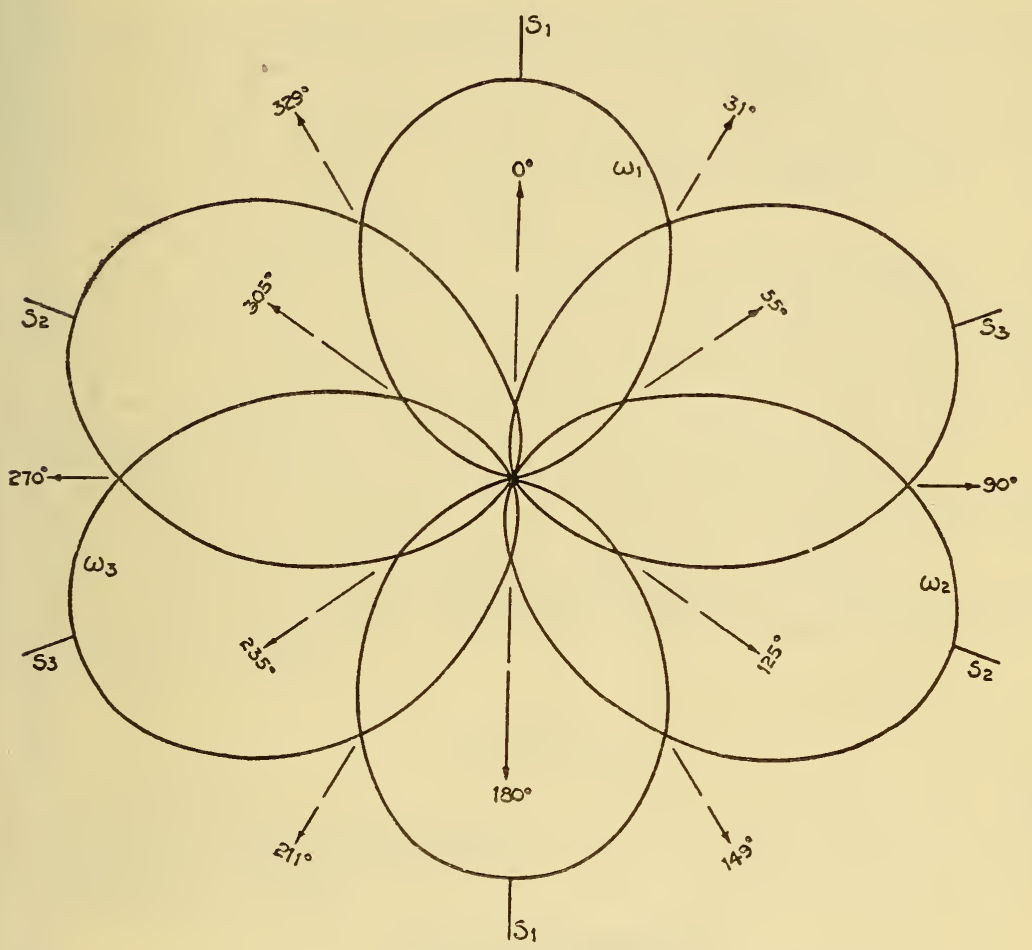

FIGURE 22.-Stator positions are the same as for Fioure 21 except that radiofrequency rather than audio-frequency switching is employed

oriented through that point by rotating the goniometer at the beacon station and the angles between the courses thus determined. These tests were repeated at a later date, the same results being obtained. These results checked also the data on the angles between courses as obtained in the air.

Washington, September 18, 1929. 